\title{
Simulation of temperature induced colorimetric shift of environmentally friendly ZnAgInSe Quantum Dots
}

\author{
Ting Ji ${ }^{1}$ a and Qi Ding ${ }^{2}$ \\ ${ }^{1}$ Tianjin Xin Hua Staff and Workers University, Tianjin, PRC 300040 \\ ${ }^{2}$ Institute of Material Physics, Tianjin University of Technology, Tianjin, PRC 300191, China \\ ajiting022@126.com
}

\begin{abstract}
Keywords: photoluminescence correlated color temperature computer simulation chromaticity coordinate ZnAglnSe Quantum Dots

Abstract. ZnAgInSe quantum dots with 5 and $6.7 \mathrm{~nm}$ particle sizes were obtained by accommodating the reaction time. The red shift in the photoluminescence spectra of ZnAgInSe quantum dots with size from 5 to $6.7 \mathrm{~nm}$ was caused by the quantum confinement effect. The temperature dependence of chromaticity coordinate and correlated color temperature of ZnAgInSe QDs with 5 and $6.7 \mathrm{~nm}$ particle size are investigated.With increasing temperature, the $\mathrm{x}$ values of CIE coordinates of ZnAgInSe QDs all increase, but z values of CIE coordinates of ZnAgInSe QDs all decrease. Correlated color temperature of ZnAgInSe QDs all increase with the temperature increase, respectively.
\end{abstract}

\section{Introduction}

Semiconductor quantum dots (QDs) have attracted considerable attention because of their specific photometric characteristics, and have been applied in light-emitting diodes, optical lasers, and photovoltaic cells [1-3]. Semiconductor QDs often refer to nanocrystals with their particle size less than twice the Bohr radius of excitons in bulk materials. At present, binary CdTe and CdSe QDs have excellent luminescent propertise comprising potentially toxic elements [4]. Development QDs comprising less toxic components are becoming the new trend. Compared to binary and ternary systems, quaternary Zn-I-III-VI nanostructures have been attracting increasing attention exhibiting tunable emissions [5]. Quaternary ZnAgInSe QDs exhibits red emission under blue excitation that is suitable to blue LED chip excitation [6]. Temperature is one of the fundamental thermodynamic state variables and the most measured physical quantity. Temperature dependent optical characteristics of QDs have been investigated [6,7]. However, low temperature dependent chromaticity coordinate and correlated color temperature of ZnAgInSe QDs with different size are seldom discussed previously. In this paper, the bandgap of quaternary ZnAgInSe QDs was adjusted by changing their size. Temperature dependence of chromaticity coordinate and correlated color temperature of ZnAgInSe QDs with different size are investigated.

\section{Experiment}

The fabrication process of the $\mathrm{Zn}(\mathrm{OAc})_{2}, \operatorname{In}(\mathrm{OAc})_{3}, \mathrm{AgOAc}$ and Se precursor solution in detail was reported in previous reference [6]. To synthesis of $\mathrm{ZnAgInSe} Q \mathrm{QDs}$, the $\mathrm{Zn}(\mathrm{OAc})_{2}, \operatorname{In}(\mathrm{OAc})_{3}$, and AgOAc precursor solutions, DDT $(2 \mathrm{~mL})$, and ODE $(6 \mathrm{~mL})$ were added into a four-neck flask. Second, the reaction mixture was slowly heated to $180^{\circ} \mathrm{C}$ under argon. Third, when the reaction mixture became clear, the Se precursor solution was injected into the flask, and the temperature was maintained at $220^{\circ} \mathrm{C}$ for $30 \mathrm{~min}$, affording $\mathrm{Zn}_{\mathrm{x}} \mathrm{AgInSe}$ QDs. Then the reaction was immediately terminated by injecting the product into toluene. The untreated $\mathrm{Zn}_{\mathrm{x}} \mathrm{AgInSe}$ QDs were purified thrice by centrifugation with methanol and acetone. Absorption spectra were recorded on a Hitachi UV-4100 spectrophotometer. Photoluminescence (PL) spectra were recorded on a Jobin Yvon FluoroLog-3 fluorescence spectrometer, and a 450-W xenon lamp was used as the light source. 


\section{Results and Discussion}

The normalized PL emission spectra and absorption spectra of ZnAgInSe QDs synthesized with different particle sizes are plotted in Figs. 1(a) and 2(b), respectively. Due to quantum confinement effect, both the absorption and emission spectra of ZnAgInSe QDs show obvious red shift from 647 $\mathrm{nm}$ to $694 \mathrm{~nm}$ with the increase of particle size from $5 \mathrm{~nm}$ to $6.7 \mathrm{~nm}$.
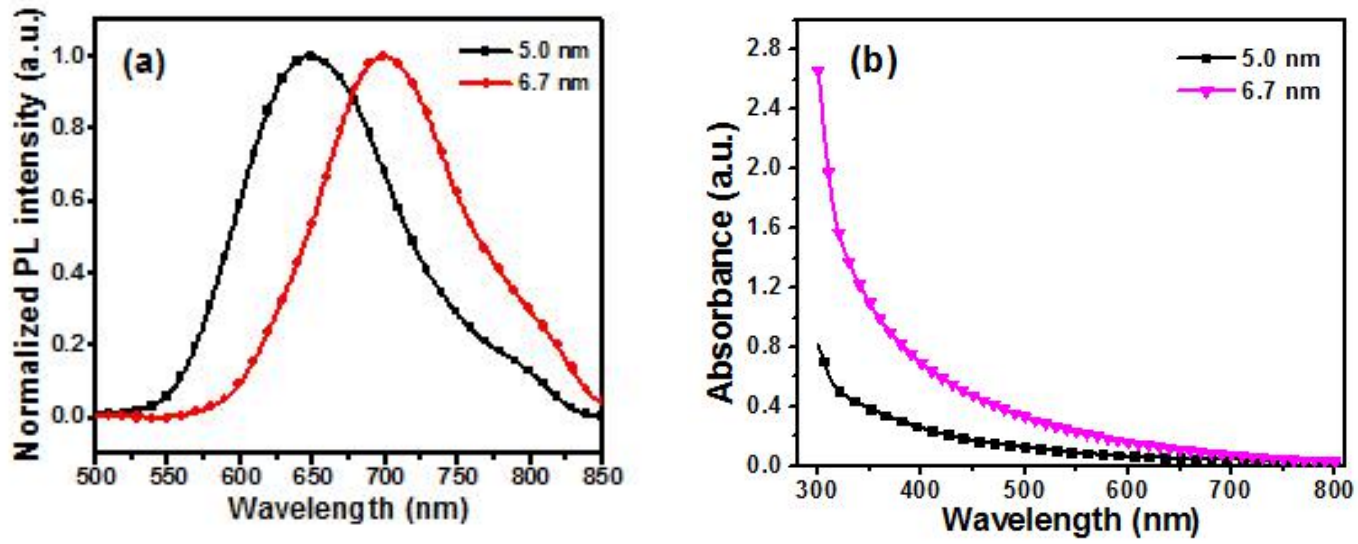

Fig.1. (a) Normalized PL emission spectra and (b) UV-vis of ZnAgInSe QDs recorded with 5 and $6.7 \mathrm{~nm}$ particle size.

Temperature dependence PL measurements of ZnAgInSe QDs with 5 and $6.7 \mathrm{~nm}$ particle sizes were measured respectively shown in Fig.2 (a) and (b). The PL intensity was found to decrease with increasing temperature from $10 \mathrm{~K}$ to $280 \mathrm{~K}$. The linewidth broadening and red shift of ZnAgInSe QDs with 5 and $6.7 \mathrm{~nm}$ in spectra were also observed.
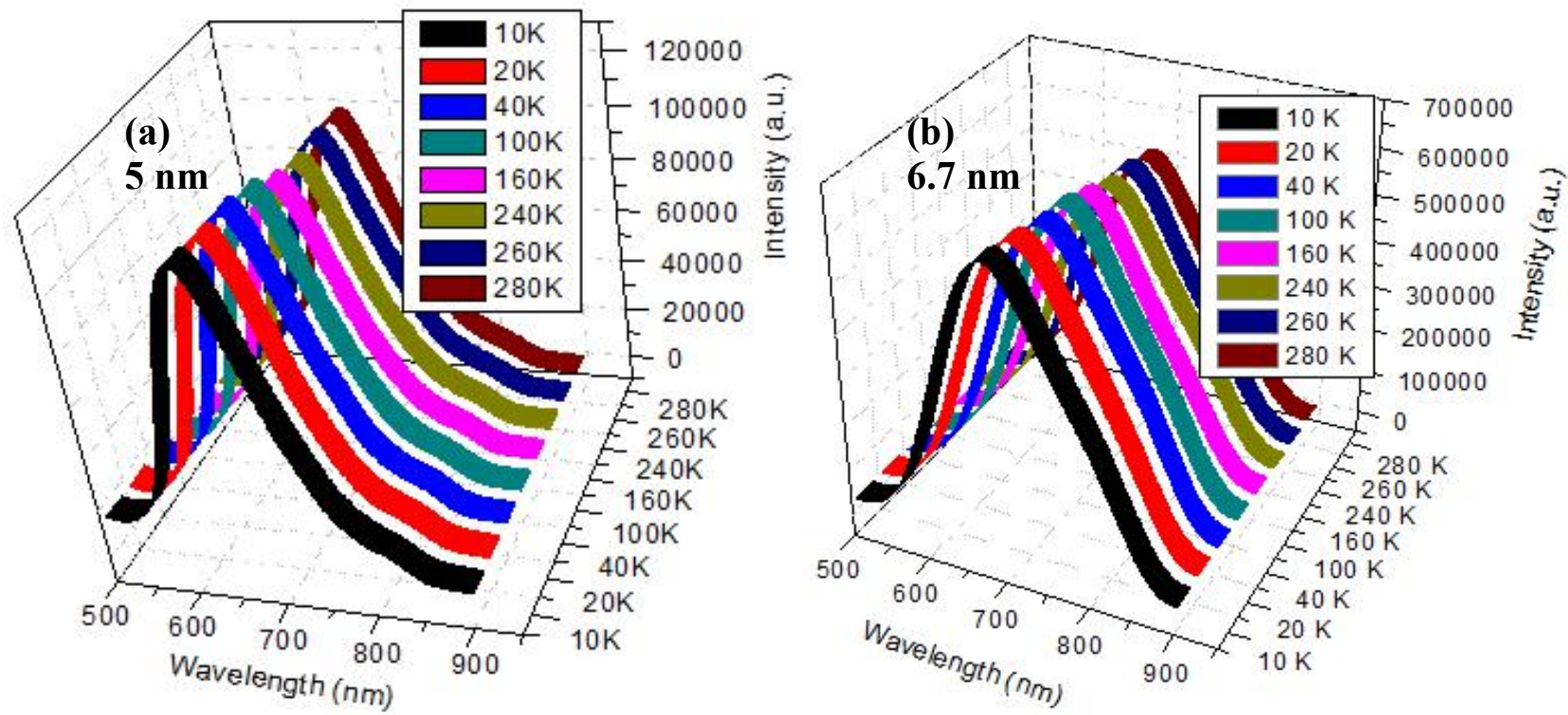

Fig.2. Temperature-dependent PL spectra of ZAISe QDs with different particle sizes (a) $5.0 \mathrm{~nm}$; (b) $6.7 \mathrm{~nm}$.

The chromaticity coordinate could be calculated using standard equations [8];

$x=\frac{\sum P(\lambda) \bar{x}_{\lambda}}{\sum P(\lambda) \bar{x}_{\lambda}+\sum P(\lambda) \bar{y}_{\lambda}+\sum P(\lambda) \bar{z}_{\lambda}}, \quad y=\frac{\sum P(\lambda) \bar{y}_{\lambda}}{\sum P(\lambda) \bar{x}_{\lambda}+\sum P(\lambda) \bar{y}_{\lambda}+\sum P(\lambda) \bar{z}_{\lambda}}, \quad z=1-x-y$, 
where the $P(\lambda)$ represents the function of spectral energy distribution, $\bar{x}_{\lambda}, \bar{y}_{\lambda}, \bar{z}_{\lambda}$ represent the color matching function, $\mathrm{x}, \mathrm{y}$ represent the $\mathrm{CIE}(\mathrm{x}, \mathrm{y})$ coordinates, and $\mathrm{z}$ represents the third chromaticity value.

The simulated chromaticity coordinate and correlated color temperature (CCT) of ZnAgInSe QDs with $5 \mathrm{~nm}$ particle size are calculated with different temperature in Table 1. Correspondingly, the chromaticity coordinates of $5 \mathrm{~nm} \mathrm{ZnAgInSe}$ QDs with different temperature are depicted in chromaticity coordinate diagram shown in Fig. 3. With temperature increasing, the $\mathrm{x}$ values of CIE coordinates increase from 0.492 to 0.523 , and y values of CIE coordinates increase to 0.328 and then decrease to 0.324 . Moreover, the $\mathrm{z}$ values as the third chromaticity value decrease from 0.188 to 0.153. With temperature increasing, the CCT value $5 \mathrm{~nm} \mathrm{ZnAgInSe}$ QDs increase from 2137 to 2889K.

Table 1 Simulated chromaticity coordinate of ZnAgInSe $(5.0 \mathrm{~nm})$ quantum dots

\begin{tabular}{|l|c|c|c|c|}
\hline & CCT [K] & $x$ & $y$ & $z$ \\
\hline $10 \mathrm{~K}$ & 2137 & 0.492 & 0.320 & 0.188 \\
\hline $20 \mathrm{~K}$ & 2189 & 0.497 & 0.322 & 0.181 \\
\hline $40 \mathrm{~K}$ & 2290 & 0.504 & 0.324 & 0.172 \\
\hline $100 \mathrm{~K}$ & 2497 & 0.517 & 0.328 & 0.155 \\
\hline $160 \mathrm{~K}$ & 2588 & 0.520 & 0.328 & 0.152 \\
\hline $240 \mathrm{~K}$ & 2601 & 0.515 & 0.324 & 0.161 \\
\hline $260 \mathrm{~K}$ & 2694 & 0.515 & 0.322 & 0.161 \\
\hline $280 \mathrm{~K}$ & 2889 & 0.523 & 0.324 & 0.153 \\
\hline
\end{tabular}

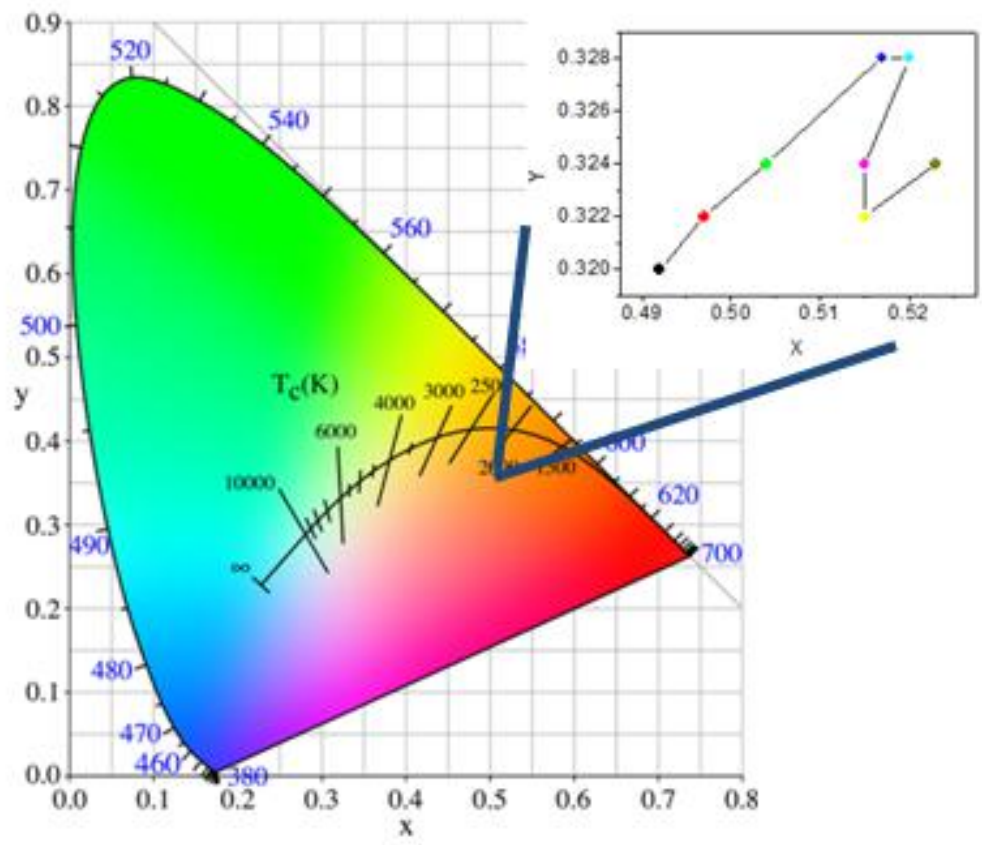

Fig.3. Temperature dependence of the chromaticity coordinate of ZnAgInSe QDs with $5 \mathrm{~nm}$ particle size.

The simulated chromaticity coordinate and correlated color temperature (CCT) of ZnAgInSe QDs with $6.7 \mathrm{~nm}$ particle size are calculated with different temperature in Table 2. Correspondingly, the chromaticity coordinates of $6.7 \mathrm{~nm} \mathrm{ZnAgInSe}$ QDs with different temperature are depicted in chromaticity coordinate diagram shown in Fig. 3. With temperature increasing, the $\mathrm{x}$ values of CIE coordinates increase from 0.515 to 0.581 , and y values of CIE coordinates increase from 0.322 to 0.355 . Moreover, the $\mathrm{z}$ values as the third chromaticity value decrease from 0.163 to 0.065 . With 
temperature increasing, the CCT value $6.7 \mathrm{~nm}$ ZnAgInSe QDs increase from 2694 to 3399K.

Table 2 Simulated chromaticity coordinate of ZnAgInSe $(6.7 \mathrm{~nm})$ quantum dots

\begin{tabular}{|l|c|c|c|c|}
\hline & CCT [K] & $\mathrm{x}$ & $\mathrm{y}$ & $\mathrm{z}$ \\
\hline $10 \mathrm{~K}$ & 2694 & 0.515 & 0.322 & 0.163 \\
\hline $20 \mathrm{~K}$ & 2952 & 0.566 & 0.353 & 0.080 \\
\hline $40 \mathrm{~K}$ & 3165 & 0.572 & 0.353 & 0.075 \\
\hline $100 \mathrm{~K}$ & 3338 & 0.575 & 0.352 & 0.073 \\
\hline $160 \mathrm{~K}$ & 3128 & 0.571 & 0.353 & 0.075 \\
\hline $240 \mathrm{~K}$ & 3185 & 0.574 & 0.354 & 0.072 \\
\hline $260 \mathrm{~K}$ & 3399 & 0.581 & 0.355 & 0.064 \\
\hline $280 \mathrm{~K}$ & 3399 & 0.581 & 0.355 & 0.065 \\
\hline
\end{tabular}

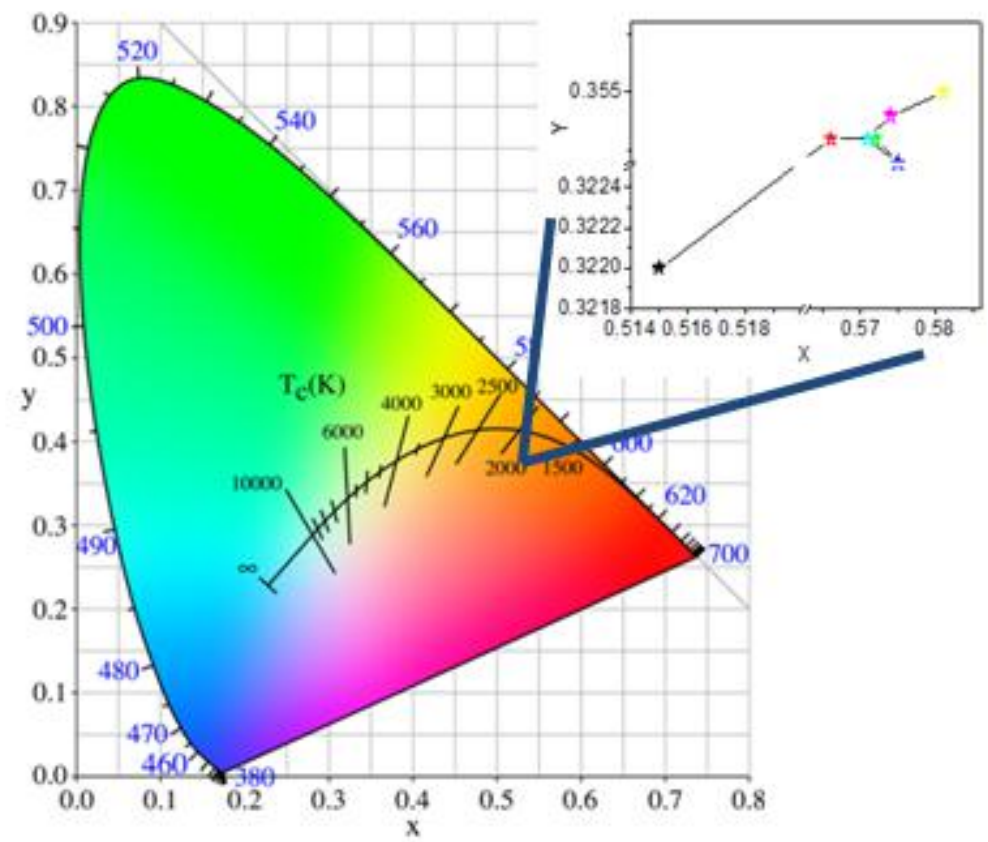

Fig.4. Temperature dependence of the chromaticity coordinate of ZnAgInSe QDs with $6.7 \mathrm{~nm}$ particle size.

\section{Conclusion}

The temperature dependences of photoluminescence properties of ZnAgInSe QDs with 5 and $6.7 \mathrm{~nm}$ particle size are investigated. Due to quantum confinement effect, both the absorption and emission spectra of ZnAgInSe QDs show obvious red shift from $647 \mathrm{~nm}$ to $694 \mathrm{~nm}$ with the increase of particle size from $5 \mathrm{~nm}$ to $6.7 \mathrm{~nm}$. Temperature dependence PL measurements of ZnAgInSe QDs with 5 and $6.7 \mathrm{~nm}$ particle sizes were measured respectively. With increasing temperature, the $\mathrm{x}$ values of CIE coordinates of ZnAgInSe QDs all increase, but z values of CIE coordinates of ZnAgInSe QDs all decrease. Correlated color temperature of ZnAgInSe QDs all increase with the temperature increase, respectively.

\section{Acknowledgements}

This work was financially supported by the Tianjin Natural Science Foundation (15JCYBJC16800, 15JCYBJC16700), National Natural Science Foundation of China (No.11504266, 51702235). 


\section{References}

[1] J. Lee, D. Wong, J. Velasco, J. F. Rodriguez-Nieva, S. Kahn, H. Z. Tsai, T. Taniguchi, K. Watanabe, A. Zettl and F. Wang: Nat. Phys. Vol.12 (2016), p.1032

[2] I. L. Medintz, H. T. Uyeda, E. R. Goldman and H. Mattoussi: Nat. Mater. Vol.4 (2005), p.435-446

[3] F. Meinardi, H. McDaniel, F. Carulli, A. Colombo, K. A. Velizhanin, N. S. Makarov, R. Simonutti, V. I. Klimov and S. Brovelli: Nat. Nanotechnology Vol.10 (2015), p.878-885

[4] Y. C. Li, H. Z. Zhong, R. Li, Y. Zhou, C. H. Yang and Y. F. Li: Adv. Funct. Mater. Vol.16 (2006), p.1705-1716

[5] X. J. Liu, X. S. Zhang, L. Li, X. L. Wang and L. L. Yuan: Chinese Phys. B Vol. 23(2014), p.117804

[6] Qi Ding, Xiao-Song Zhang, Lan Li, Jian-Ping Xu, Ping Zhou, Xiao-Fei Dong, and Ming Yan: Chin. Phys. B Vol. 26 (2017), p. 67804

[7] P. Zhou, X. S. Zhang, X. J. Liu, J. P. Xu and L. Li: Opt. Express Vol. 24 (2016), p.19506-19516

[8] G. Wyszecki and W. Stiles, in: Color Science -- Concepts and Methods, Quantitative Data and Formulate (2nd Edition), Wiley, New York (1982). 